\title{
Optimization of the Data Traffic Distribution based on Hierarchical Mobile Networks
}

\author{
Ronnie D. Caytiles \\ Multimedia Engineering Department, Hannam University \\ 133 Ojeong-dong, Daeduk-gu, Daejeon, Korea \\ rdcaytiles@gmail.com
}

\begin{abstract}
Data traffic distribution across heterogeneous mobile networks requires a control management in order to optimize the handoffs between its entities. The more the technologies are evolving, the more complex the distribution of data traffic becomes. The standard MIPv6 and hierarchical MIPv6 mobility management were introduced in order to address the flow mobility of data traffic. This paper exploits the multi-connectivity option for mobile nodes in HMIPv6 in order to utilize the optimum route for the data traffic distribution.
\end{abstract}

Keywords: HMIPv6, Multi-connectivity, traffic distribution, flow mobility

\section{Introduction}

Recently, the Internet Engineering Task Force (IETF) has standardized a number of protocols in order to address the uprising issues on communications of mobile devices across the heterogeneous networks. The Mobile Internet Protocol version 6 (MIPv6) was standardized and is capable of providing mobile nodes a continuous connection even if it frequently moves its location [1]. The Hierarchical Mobile Internet Protocol version 6 (HMIPv6) was released as an enhanced MIPv6 to address the issues concerning the high handover latency of the former due to a substantial number of required signaling for the establishment of connection of mobile devices and its correspondent nodes [3].

The HMIPv6 is designed to limit the amount of signaling between the mobile node, correspondent nodes, and its home agent for MIPv6 by introducing a new concept of adding a Mobility Anchor Point (MAP) that acts as a local home agent (central controller). The MAP is responsible for controlling the Access Routers (RAs) that are available within the HMIPv6 domain. However, the available ARs have varying handoff latencies to which is depending on the bandwidth, traffic conditions, congestions due to a number of connected nodes, routing issues, etc. Thus, to optimize the routability and data traffic distribution among mobile nodes, the multi-connectivity is exploited in order to provide an efficient flow mobility management.

This paper deals with the management of multi-connectivity capability of mobile nodes based on HMIPv6. The process for the selection of a particular AR to establish the point of attachment by a mobile node is presented in order to address or somehow limit the issues with regards to higher handover latency. The MAP as the central controller determines the current traffic conditions as the basis for the $\mathrm{MN}$ to select between the most optimized handover between various access routers (ARs) within the HMIPv6.

The rest of this paper is organized as follows: Section 2 explains the basic operations and the standard handover procedure of the HMIPv6; Section 3 discusses the data traffic distribution based on hierarchical mobile networks; and the concluding remarks in Section 4. 


\section{Overview of Mobile Networks}

The capability of the IETF mobility standard Mobile Internet Protocol version 6 (MIPv6) to maintain the connectivity for a mobile node (MN) with the correspondent nodes while it moves within the MIPv6 domain requires a lot of signaling or sending of binding updates $[1,2,5]$. The $\mathrm{MN}$ establishes its new connection by sending binding updates to its home agent (HA) as well as to all the correspondent nodes (CNs) that the $\mathrm{MN}$ wishes to communicate with. Every time the MN moves to another subnet location, it disconnects its attachment and establishes a new connection. As a result, substantial messaging is required that provides additional delay and increased handover latency. Thus, data traffic distribution for MNs is constrained by the time required for processing its disconnection and re-connection every time that it moves to a different subnet location.

In order to compensate the lost time of sending a number of signaling for reestablishment of MN's connectivity, the IETF has proposed an enhancement protocol for MIPv6 that utilizes a new Mobile IPv6 node, called the Mobility Anchor Point (MAP), located at any level in a hierarchical network of routers, including the Access Router (AR). The MAP aims to manage the local handoffs and reduce the amount of Mobile IPv6 signaling outside the local domain [3,5]. This enhancement protocol is referred to as Hierarchical Mobile Internet Protocol version 6 (HMIPv6) and help improve the handoff procedure speed by reducing the amount of signaling $[4,6]$. The local and global mobility for HMIPv6 were separated to which the local handovers are handled locally.

In HMIPv6, the binding updates are sent by the MN to the MAP instead of sending it directly to the HA and CNs. After the connection is established, data traffic intended to the MN from its home agent and CNs are re-routed by the MAP to its current location through a bidirectional tunnel created between the MN and the MAP. The MAP acts as the central controller or an intermediary to which it directs the traffics from the HA and all CNs to the particular $\mathrm{MN}$ regardless of its current location.

HMIPv6 starts its operations whenever a MN enters a MAP domain. The MN will receive Router Advertisements (RAs) containing information regarding the available local MAPs. The current location of MN is then configured, on-link Care-of address (LCoA), with the regional Care-of address (RCoA) which is an address on the MAP's subnet. The Regional Care-of Address (RCoA) is an address on the MAP's subnet based on the prefix in the MAP option of the RA message sent by MAP. It is an address obtained by the mobile node from the visited network. It is auto-configured by the MN when receiving the MAP option.

On the other hand, the On-link CoA (LCoA) is an address configured on an MN's interface based on the prefix advertised by its default router (AR). As soon as the two CoAs are configured, the MN sends a BU message to the HA and CNs through the MAP. A bidirectional tunnel will be created between the MN and the MAP as soon as the binding acknowledgements (BA) have been received. The MAP then receives all packets on behalf of the MN and will encapsulate and forward them directly to the mobile node's current address instead of the HA (wherein the MAP is acting as a local HA).

Whenever the MN moves its location within the same MAP domain (LCoA), it will only send the BU message to the MAP to register the new address. Thus, only the regional $\mathrm{CoA}(\mathrm{RCoA})$ needs to be registered with correspondent nodes and the HA as in Figure 1. However, the RCoA does not change as long as the MN moves within a MAP domain, thus making the mobile node's mobility transparent to the $\mathrm{CN}$ where it exchanges signals with. 


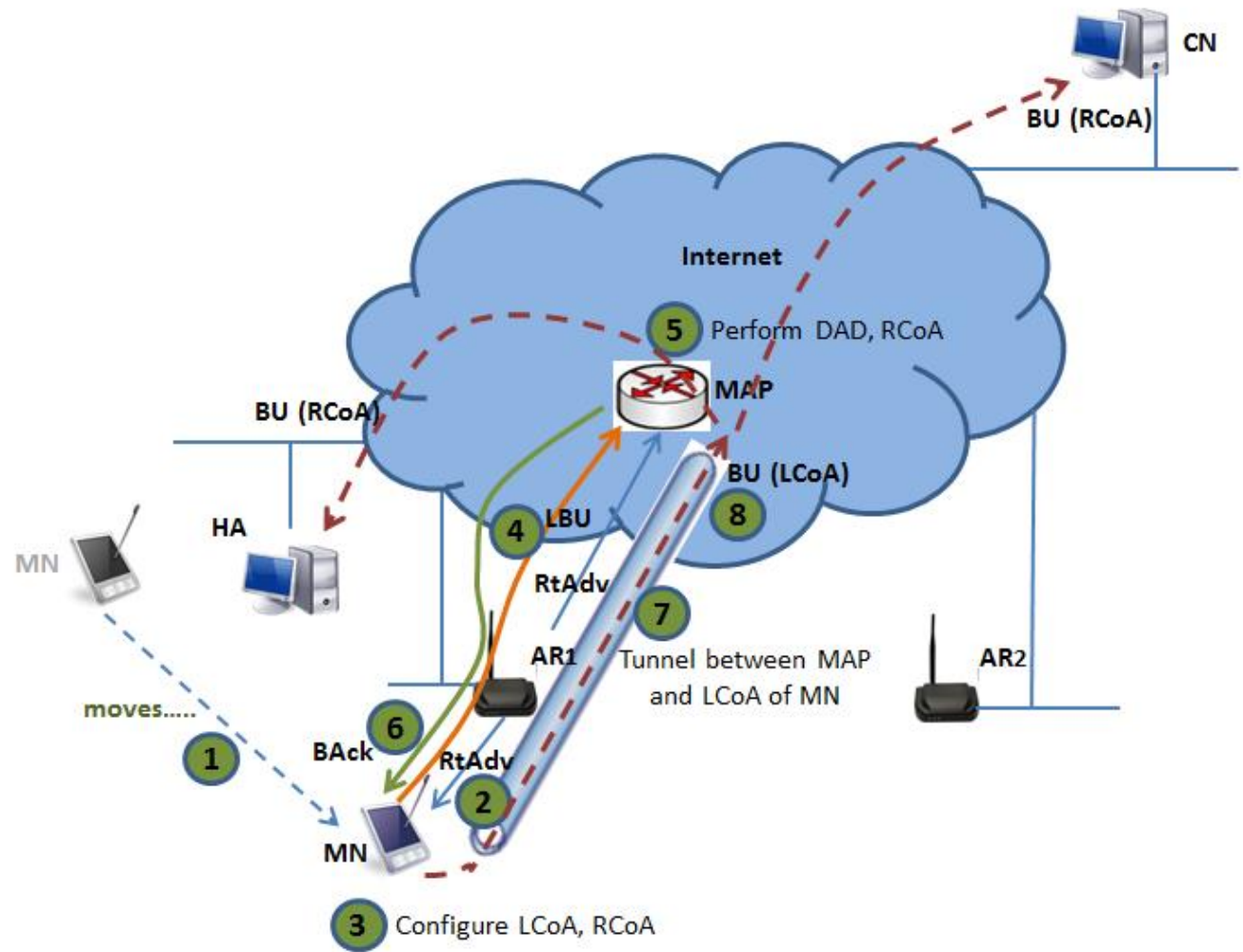

Figure 1. Hierarchical Mobile IPv6 Flow Mobility Operations

Whenever the MN moved from AR1 to AR2 in Figure 1, the movement is transparent to the HA and all CNs, thus it is only required to configure only the New LCoA and bind with MAP. The tunnel is then moved between MAP and AR2.

\section{Data Traffic Distribution based on Hierarchical Mobile Networks}

Multi-connectivity for MNs paves the way for data traffic distribution across different ARs that are located within the HMIPv6 domain. The MN is capable of making multiple connectivity across the available ARs to which have a varying traffic and connectivity status on a heterogeneous mobile environment. In a particular scenario wherein the MN can be located between co-existing ARs, the MN has the capability of choosing the point of connection that could provide the optimum handover latency for receiving data traffic from a particular $\mathrm{CN}$. This is practically important whenever $\mathrm{CNs}$ is located to a specific location within a particular AR domain with a strong signal. The MAP will be responsible to determine the traffic information on the collection of possible routes that will result on the MN to switch between multiple ARs in order to optimize the data traffic distribution. 


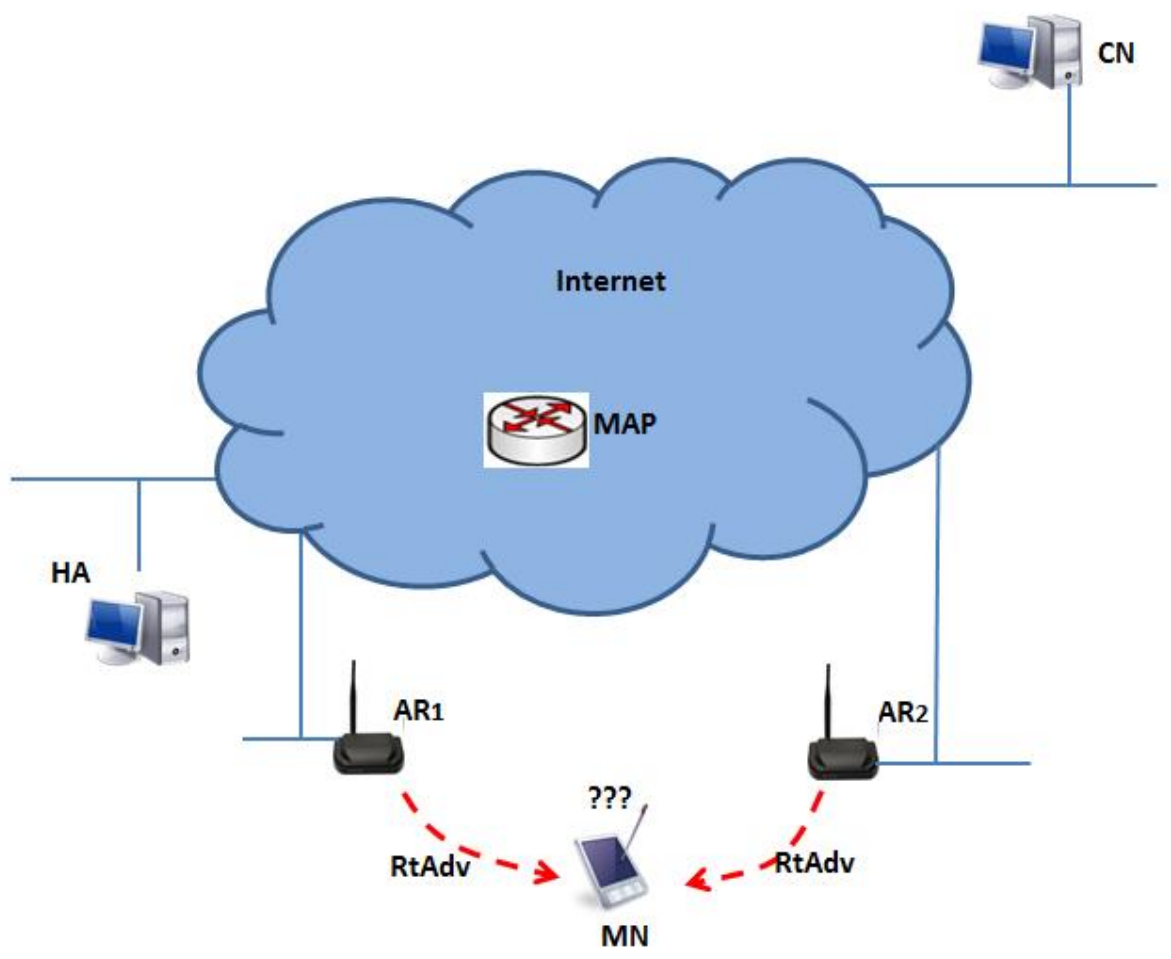

Figure 2. MN is Capable of Switching between Available ARs

Figure 2, depicts an MN that is able to choose between available ARs. In this study, the MN is assumed to be connected in one of the ARs available in the HMIPv6 domain wherein the MN's LCoA has been configured to the MAP and RCoA was also configured in the $\mathrm{HA}$ as well as to all the CNs that the MN is communicating. The data traffic intended to the MN will be intercepted by the MAP and will be encapsulated and re-routed whichever path (chosen AR) has the optimized handover latency.

By acquiring traffic information of all available ARs in the MAP domain, the MN can be allowed to switch between ARs and thus optimizing the handoff process. The MAP sends a flow control signaling to all available ARs in order to determine the necessary traffic information for the purpose of identifying the optimized path for the distribution of data traffic across hierarchical mobile networks as in Figure 3. The ARs that have received the flow signaling message replies with an acknowledgement that contains the traffic information, i.e., handover latency, bandwidth, traffic congestions based on the number of connected nodes, etc. As soon as the optimized route is determined, the $\mathrm{MN}$ sends a binding update in order to configure its LCoA to the MAP. The bidirectional tunnel is then moved to the AR wherein the $\mathrm{MN}$ attaches its new point of connection. This flow control signaling will be sent periodically in order to maximize the possibility of acquiring the best route for the distribution of network traffic. 


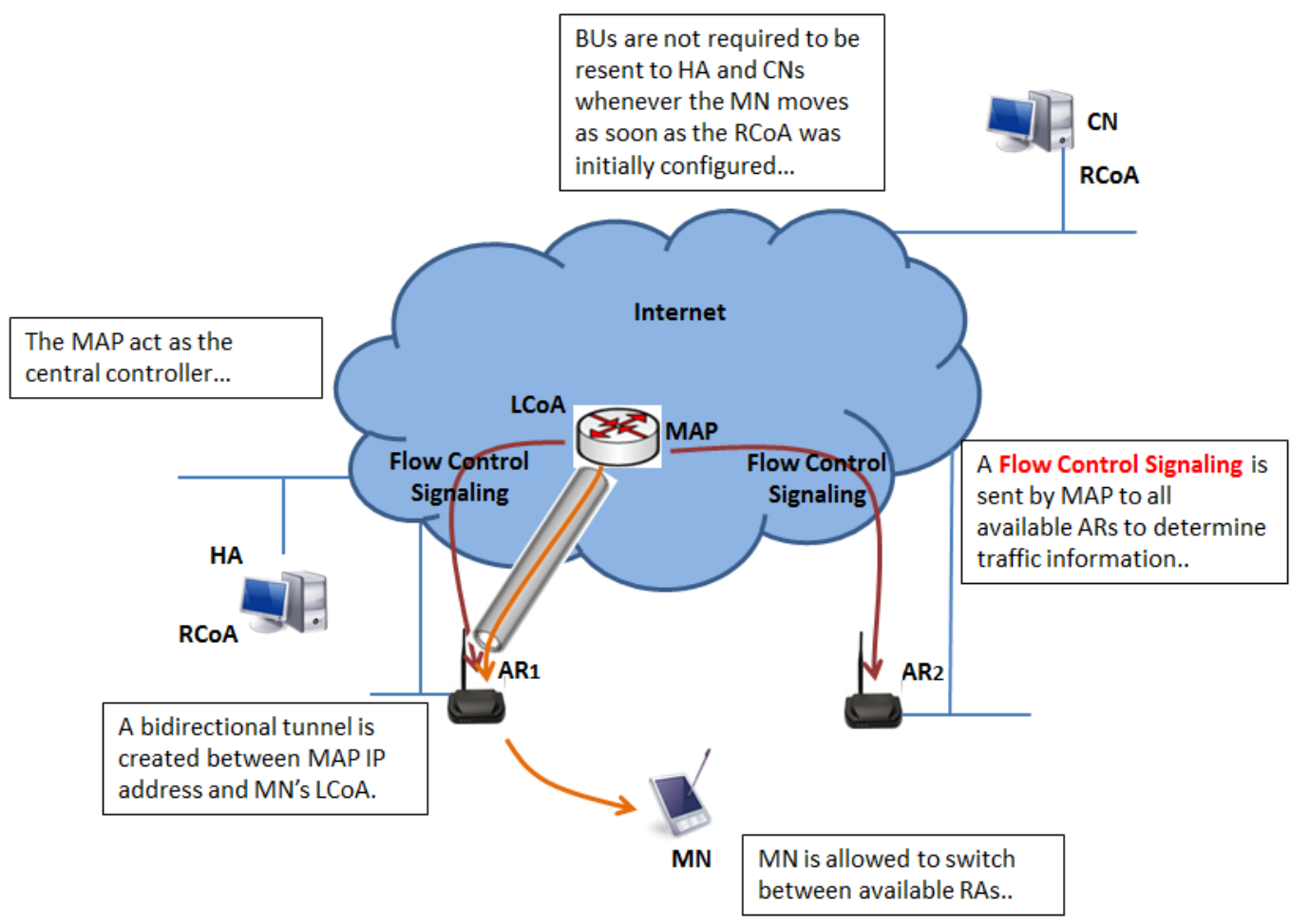

Figure 3. Flow Control Signaling is sent by the MAP in order to Control the Movement of MN and Distribute Data Traffic

The RCoA is not required to be configured anymore and the MAP will be responsible for intercepting all data traffic packets intended to the MN. Data traffic will be encapsulated and delivered through the bidirectional tunnel that is established between the MAP's IP address and the MN's LCoA. In this regard, the local mobility will be managed by the MAP.

In the case that a particular $\mathrm{CN}$ that wanted to send data traffic to the $\mathrm{MN}$ is located within one of the AR with a stronger signal, the data traffic could be distributed efficiently without any delays. The ARs with weaker signals can only be utilized if congestion and bandwidth for ARs with stronger signals are not good.

\section{Conclusion and Future Works}

This paper has presented an efficient data traffic distribution management based on heterogeneous mobile networks. The multi-connectivity for mobile nodes were exploited in order to efficiently distribute the data traffic and provide a centralized control over the optimization of handoffs between the mobile node and the mobility anchor point (MAP). The best route for distributing data traffic can be selected in order to enhance the handover performance for HMIPv6. In the future, the computation of the traffic information that are determined by the flow control signaling will be shown and analyzed in order to further exploit the multi-connectivity for mobile devices.

\section{Acknowledgments}

This paper has been supported by the 2015 Hannam University Research Fund. 


\section{References}

[1] D. Johnson, C. Perkins, et al., "Mobility Support in IPv6", Internet Engineering Task Force (IETF), Request For Comment (RFC) 3775, (2004) June.

[2] C. Perkins, D. Johnson and J. Arkko, "Mobility Support in IPv6", Internet Engineering Task Force (IETF), Request For Comment (RFC) 6275, ISSN: 2070-1721, (2011).

[3] H. Soliman, C. Castelluccia, K. ElMalki and L. Bellier, "Hierarchical Mobile IPv6 (HMIPv6) Mobility Management”, IETF, RFC No. 5380, http://www.rfc-base.org/txt/rfc-4140.txt, (2008) October.

[4] http://searchmobilecomputing.techtarget.com/definition/Hierarchical-Mobile-IPv6.

[5] X. Pérez-Costa, M. Torrent-Moreno and H. Hartenstein, "A performance comparison of Mobile IPv6, Hierarchical Mobile IPv6, fast handovers for Mobile IPv6 and their combination" ACM SIGMOBILE Mobile Computing and Communications Review, vol. 7, no. 4, (2003), pp. 5-19.

[6] X. Pérez-Costa and M. Torrent-Moreno, "A performance study of hierarchical mobile IPv6 from a system perspective", IEEE International Conference on Communications 2003, ICC'03, vol. 1, IEEE, (2003). 